\title{
James T. Richardson, François Bellanger (eds.), Legal Cases, New religious Movements and Minority Faith
}

Farnham, Ashgate, coll. « Ashgate Inform Series on Minority Religions and Spiritual Movements ", 2014, 278 p.

Bibiographie, index.

\section{Régis Dericquebourg}

\section{CpenEdition} Journals

\section{Édition électronique}

URL : http://journals.openedition.org/assr/27446

DOI : 10.4000 /assr.27446

ISSN : $1777-5825$

Éditeur

Éditions de l'EHESS

Édition imprimée

Date de publication : 1 octobre 2015

Pagination : 358

ISBN : 978-2-7132-2515-4

ISSN : 0335-5985

Référence électronique

Régis Dericquebourg, « James T. Richardson, François Bellanger (eds.), Legal Cases, New religious Movements and Minority Faith », Archives de sciences sociales des religions [En ligne], 172 I octobredécembre, mis en ligne le 09 juin 2016, consulté le 24 septembre 2020. URL : http:// journals.openedition.org/assr/27446; DOI : https://doi.org/10.4000/assr.27446

Ce document a été généré automatiquement le 24 septembre 2020.

(c) Archives de sciences sociales des religions 


\section{James T. Richardson, François Bellanger (eds.), Legal Cases, New religious Movements and Minority Faith}

Farnham, Ashgate, coll. « Ashgate Inform Series on Minority Religions and Spiritual Movements ", 2014, 278 p.

Bibiographie, index.

Régis Dericquebourg

\section{RÉFÉRENCE}

James T. Richardson, François Bellanger (eds.), Legal Cases, New religious Movements and Minority Faith, Farnham, Ashgate, coll. « Ashgate Inform Series on Minority Religions and Spiritual

Movements ", 2014, 278 p. 
1 La multiplication des groupes religieux minoritaires ou tout simplement la plus grande visibilité que les plus anciens ont acquise depuis les années 1960 - d'abord dans les pays occidentaux, puis à un niveau mondial - a provoqué des réactions négatives envers eux chez des opposants déclarés à toute religion qui y trouvaient un surplus d'irrationnel, ou chez des fidèles des Églises établies qui craignaient la concurrence sur un marché des biens du salut qu'elles prétendaient monopoliser. Les pressions que les premiers et les seconds ont exercées sur les politiciens ont conduit plusieurs gouvernements à harceler les groupes religieux minoritaires (sectes, groupes métaphysiques, «cult», ordres initiatiques... parfois baptisés
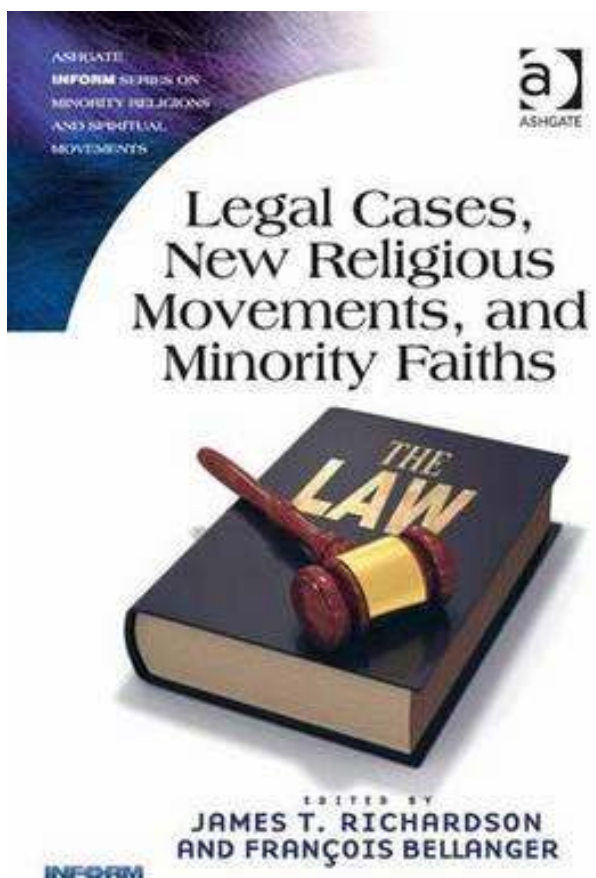
uniformément de secte, au sens péjoratif), en invoquant des risques pour l'individu, pour la famille ou pour l'ordre public. De ce fait, des pratiques liées au harcèlement ont été présentées aux juges par les deux adversaires. Le harcèlement juridique fait partie de la stratégie des opposants aux groupes religieux minoritaires et de certains gouvernements. En contrepartie, les mouvements religieux qui disposent des finances nécessaires assignent leurs détracteurs en justice. Ceci a été consigné dans une littérature où l'on trouve des analyses d'avocats, des interprétations de chercheurs, de commentaires de groupes religieux minoritaires et de leurs opposants. La production est abondante en France qui est un des pays occidentaux qui a porté le plus loin la lutte contre les dites sectes, jusqu'aux professeurs de yoga affublés du titre de gourou. Le livre que nous présentons appartient à cette veine. Il constitue les actes d'un colloque organisé par l'association anglaise «Inform » (qui a pour objet de réunir des études objectives sur les minorités religieuses et de les faire connaître). Il est publié dans la collection "Inform On Minority Religions and Spiritual Movements». La direction de l'ouvrage a été confiée à James T. Richardson et François Bellanger, spécialistes du Droit des minorités religieuses. Ils ont rassemblé des analyses d'universitaires, de nonuniversitaires et de personnes qui ont un discours critique envers les groupes religieux minoritaires.

D'un point de vue général, le livre porte sur des procès qui mettent en question des principes religieux ou institutionnels et des pratiques.

Parmi les grands principes, il y a ceux des Droits de l'enfant et la lutte contre la «maltraitance» des mineurs. Claire Borowik rappelle l'affaire des raids de la gendarmerie contre les communautés de la Famille (ex-Enfants de Dieu) dont un en France (à Eguilles) sur le conseil d'associations antisectes expertes autoproclamées en « sectes ». La justice a conclu à l'innocence des fidèles mais l'affaire montre la fragilité du lien entre la protection du bien-être des enfants et la liberté religieuse. Tirant les leçons de ce type d'opération qui a eu lieu simultanément dans plusieurs pays 
occidentaux, la magistrate australienne Gregory Levine (1993), comme ses collègues argentins, a mis en garde les autorités contre l'utilisation des droits de l'enfant dans le harcèlement des petites religions. Dans l'affaire de la Famille, les autorités auraient franchi la ligne rouge car ils n'avaient pas évalué les dommages des raids sur les enfants à l'exception de l'Australie qui les a indemnisés. À une époque où on met en place des «cellules psychologiques " lors de catastrophes et d'accidents, aucune prise en charge des enfants choqués par le raid et l'éloignement des parents n'a été proposée. La question de la protection de l'enfant est également posée par Monica K. Miller à propos des Églises de guérison. Le droit à la liberté religieuse (tel qu'il est par exemple reconnu par la Constitution américaine) peut-il s'appliquer à ceux qui revendiquent, au nom de leur foi, de remplacer un traitement médical par la prière ? La question a des implications réelles puisqu'on estime à 172 le nombre d'enfants décédés à cause d'un recours au «Faith healing » seul entre 1975 et 1995 aux États-Unis. Naturellement, le livre examine le cas de l'Église de la science chrétienne qui est la plus organisée dans l'administration du traitement spirituel. Globalement, la convention des Nations Unies sur les droits de l'enfant permet aux parents de choisir le traitement qu'ils souhaitent pour guérir l'enfant. Toutefois, elle est complétée par la décision d'Ottawa sur les droits de l'enfant qui stipule qu'un traitement médical doit être appliqué si l'enfant est en danger de mort. Ainsi, la science chrétienne qui avait fait l'objet d'une tolérance particulière a été soumise à l'obligation de soins en 1983 après le décès d'un enfant malade dans ses rangs. Une seconde catégorie de procès porte sur le droit au respect des croyances et des pratiques (Mullis A. \& Scott A.). La loi sur la diffamation concerne d'abord les individus et elle repose sur la reconnaissance de l'importance de la réputation. Pour les juges, il faut que la diffamation soit juridiquement constituée. Toutefois, les auteurs constatent une généralisation des plaintes liées aux critiques des doctrines et des pratiques ainsi qu'aux accusations d'immoralité. Là encore, il est difficile de marquer la frontière entre la critique des religions qui est un usage de la liberté d'expression et la diffamation. Le tribunal apprécierait les commentaires sur les religions selon leur "intérêt général » et selon la bonne foi de l'auteur. En somme, le propos ne doit pas être malveillant. Un rationaliste peut écrire que des fidèles perdent leur temps en fréquentant les services religieux sans qu'il y ait malveillance puisque le commentaire représente un avis ordinaire de libre penseur. Pour les auteurs, quand la diffamation ne concerne pas des faits irrespectueux et faux imputés à une personne, il est préférable de recourir à la négociation entre les parties plutôt qu'au juge. Le même problème est repris au chapitre 9 de l'ouvrage par Hardeep Singh. Ce dernier décrit les disputes entre les groupes de sikhs anglais. L'un d'eux s'estimait diffamé par l'autre à cause de propos sur ses croyances. Le tribunal sécularisé a décidé de ne pas intervenir dans cette querelle. Globalement, la Cour européenne des droits de l'homme a été sollicitée pour des cas relevant des Droits fondamentaux de l'homme (Lykes V. A. \& J. T. Richardson). Ils se passent en Russie et en France. Dans ces pays, le harcèlement des groupes religieux minoritaires a atteint un niveau intense. En Russie, on le doit à la pression des orthodoxes et en France on le doit à l'influence des obédiences maçonniques rationalistes et à l'Église catholique romaine. La CEDH a reconnu 40 violations de l'article 9 sur la liberté de conscience entre 1990 et 2011 pour ces pays en appliquant la jurisprudence Kokkinakis, du nom d'un Témoin de Jéhovah qui avait fait condamner la Grèce. La France a également été condamnée après la plainte des Témoins de Jéhovah (deux fois), par l'Aumisme (une fois) et par l'Église évangélique missionnaire (une fois) pour un montant de 3696005 euros de réparation du préjudice 
subi. La Russie a été sanctionnée par la CEDH pour sa procédure d'enregistrement restrictive mise en place en 1997. Pour les auteurs, une ébauche de jurisprudence pour l'Europe se dessine dans ces décisions judiciaires. La question d'une orientation prochrétienne de la CEDH a été posée, elle trouvera sa réponse dans la réponse aux recours des musulmans. Il y a un autre domaine pour lequel la justice a été sollicitée : celle de la qualification des religions. Certes les juges ne sont pas appelés à dire si un mouvement est religieux ou non mais ils peuvent accorder ou refuser des bénéfices spécifiques aux religions à un mouvement qui les demande. On en trouve un exemple en France avec le droit de recevoir des dons et des legs (loi de 1905, 1907). Massimo Introvigne examine les vingt années de procès au terme desquelles la cour d'appel de Milan a prononcé un jugement qui attribue à l'Église de la Scientologie les mêmes droits que les Églises établies. À cette occasion, la question de la définition de la religion et de la diversité des croyances a été posée ainsi que la différence entre le jugement des juges et celui des médias, la question de la gratuité ou du paiement des services religieux (on a rappelé la vente des indulgences par l'Église catholique dans le passé)... Pour Massimo Introvigne, cette décision ouvre une perspective plus libérale dans les Cours de Justice italienne pour reconnaître ou non la qualité de religion. Un autre type de recours à la justice est celui du conflit entre des pratiques spirituelles supposées et la politique d'un État. Le cas est illustré par un groupe qui revendique de consommer rituellement de la drogue dans un pays comme les États-Unis qui a déclaré la « guerre à la drogue ». Il s'agit de " O Centro Espirita Beneficiente Uniao do Vegetal» (UDV) qui comme le Holy light of the Queen utilise dans son "thé sacré » une molécule hallucinogène (le DMT) considérée comme une drogue aux États-Unis. J. T. Richardson et J. Shoemaker relatent l'affaire. Après onze années de procès, un accord est intervenu entre les autorités et l'UDV, selon lequel cette dernière peut consommer rituellement le Hoasca tea (" thé sacré ») rejoignant ainsi les Amérindiens qui ont obtenu le droit au peyote en 1990. Cet accord peut avoir des conséquences sur la politique antidrogue des États-Unis dans la mesure où les rastafaris pourraient aussi gagner le droit de consommer rituellement de la marijuana. Un autre type de recours à la justice évoqué est celui du discours révolutionnaire d'un groupe religieux dans les pays qui luttent contre le terrorisme. La justice peut-elle dire si un discours religieux radical est terroriste? Le cas a été posé autrefois aux États-Unis avec les Témoins de Jéhovah qui condamnent radicalement la "société » et qui ont été considérés récemment comme extrémistes en Russie. Susan Palmer examine le problème à partir d'un raid rocambolesque du FBI contre neuf communautés Huttaree, une milice chrétienne du Michigan en 2012. Deux ans après le raid, les membres incriminés ont été acquittés de l'accusation de terrorisme, de complot et de planification de guérilla contre la police. La question posée est celle du rapport entre une " parole libre » et la sédition réelle. Dans les faits, l'affaire a démarré à cause d'une erreur de délation d'un agent du FBI, cela fut suivi par des informations erronées données par un informateur loufoque payé pour s'insérer dans le mouvement et enfin par le mensonge du FBI qui a fait croire que l'agent infiltré était mort. De plus, le FBI a classé la Bible King's James saisie dans le groupe comme «littérature antigouvernementale». De cette manière, les Huttaree devenaient des comploteurs. Ceci ressemble au fiasco de Waco en 1993 mais celui-ci fut plus dramatique. Dans cette affaire rocambolesque, des gens ont passé deux ans en prison et ont été vilipendés par la presse. Par la suite, les Huttaree ont été adulés par des journalistes, par la gauche radicale américaine et sont devenus une légende pour les patriotes américains. D’autres chapitres du livre portent sur le fonctionnement interne 
de la justice face aux minorités. E. Moeder et J. Pfeifer passent en revue les travaux sur les stéréotypes qui affectent le jugement de culpabilité (études sur l'ethnicité et l'attribution d'une culpabilité). Ce sont en fait surtout des travaux de psychologie sociale expérimentale réalisés en laboratoire. Ils sont éloignés de la réalité d'un procès ou de la délibération de jurés où la méthodologie du jugement peut diminuer ou même annuler l'attitude préalable comme le montrent... d'autres expérimentations! Sous la plume de J. F. Mayer, l'ouvrage revient sur l'affaire dite du « suicide des adeptes du temple solaire». Les enquêteurs connaissaient le fonctionnement des criminels mais pas ceux des groupes religieux ou des Ordres initiatiques. En fait, seule une personne a été jugée: Michel Tabachnik à cause de ses écrits ésotériques qui seraient incompréhensibles. La carrière de ce chef d'orchestre a été interrompue (avant de reprendre brillamment à Bruxelles). Enfin, un autre chapitre un peu atypique porte sur ce que les universitaires peuvent dire ou taire à la justice (P. Katz) à propos de groupes religieux ou de personnages qu'ils ont étudié. Il s'appuie sur le cas de Moham Singh, maître d'un cercle bouddhiste et guérisseur qui fut mis en examen pour viol. 